\title{
Optimization of Amplify-and-Forward Multicarrier Two-Hop Transmission
}

\author{
Wenyi Zhang, Member, IEEE, Urbashi Mitra, Fellow, IEEE, and Mung Chiang, \\ Member, IEEE
}

\begin{abstract}
In this paper, frequency-domain relay processing in a two-hop transmission system is investigated. The relay is constrained to be "non-regenerative"; that is, the relay is only allowed to perform symbolby-symbol memoryless transformation of its received signals. Multicarrier modulation, e.g., orthogonal frequency division multiplexing (OFDM), is utilized to convert each hop link into a collection of noninterfering parallel subcarriers. In contrast to conventional scalar amplify-and-forward (AF) relays that scale all the subcarriers uniformly, it is possible to suppress relay noise and to exploit frequency-domain diversity by optimizing the relay scaling coefficients of different subcarriers jointly with subcarrier power allocation at the source transmitter. Although the end-to-end achievable rate is a non-concave function of the power allocation vectors, its optimization is accomplished with an algorithm whose computational complexity grows linearly with the number of subcarriers, by utilizing a structural property of the problem. Further motivated by the problem structure, a suboptimal algorithm with even lower complexity is also proposed, in which each hop link performs waterfilling separately over a selected subset of subcarriers. For hop links with a frequency-flat channel profile, the maximum achievable rate is explicitly derived as is the associated optimization. For hop links with Rayleigh fading frequencydomain channel profiles, numerical simulation results are presented, and it is illustrated that the proposed low-complexity suboptimal algorithm usually achieves near-optimal performance.
\end{abstract}

The work of W. Zhang and U. Mitra was supported in part by NSF OCE-0520324. Preliminary results in this paper have appeared in part in [1].

W. Zhang was with the Ming Hsieh Department of Electrical Engineering, University of Southern California, Los Angeles, CA, and he is now with Qualcomm Research Center, San Diego, CA. (Email: wenyizha@gmail.com).

U. Mitra is with the Ming Hsieh Department of Electrical Engineering, University of Southern California, Los Angeles, CA. (Email: ubli@usc.edu).

M. Chiang is with Department of Electrical Engineering, Princeton University, Princeton, NJ. (Email: chiangmeprinceton.edu). 


\section{Index Terms}

amplify-and-forward, multicarrier, multihop network, non-convex optimization, relay network, OFDM, rate optimization

\section{INTRODUCTION}

In a multihop transmission system, information flows through hop links connected by relays (repeaters) from a source to a destination. Such systems have been persistently studied since the early days of communication engineering; see, e.g., [2]-[6] and references therein for an incomplete list covering various aspects of multihop transmission. ${ }^{1}$ Multihop transmission dramatically benefits the per-hop signal-to-noise ratio (SNR) by splitting a long transmission range into multiple shorter hops, and thus is an indispensable solution in various systems like digital subscriber lines (DSL), wireless mesh networks, satellite networks, underwater acoustic networks, low-power sensor networks, and optical networks.

Among different processing techniques at relay nodes, the decode-and-forward (DF) scheme achieves the capacity of a multihop transmission system, as can be readily argued by a cutset bounding technique [8]. In certain practical systems, however, due to other considerations like complexity (e.g., [7]) or latency (e.g., [6]), nodes may adopt simpler signal processing techniques, in particular, the so-called non-regenerative relaying in which only symbol-by-symbol memoryless transformation is allowed. In single-carrier modulation, the non-regenerative relay processing is conventionally implemented by an amplify-and-forward (AF) scheme, in which the received signal is linearly scaled by a scaling coefficient and then transmitted toward the destination.

The scalar AF scheme fails to suppress the relay noise, and fails to exploit potential frequency diversity exhibited by the hop links, especially in multicarrier modulation like orthogonal frequency division multiplexing (OFDM) which decomposes each hop link into a collection of noninterfering parallel subcarriers. In this paper, we investigate the problem of jointly optimizing the relay AF scaling coefficient for each subcarrier in addition to the subcarrier power allocation at the source transmitter. For tractability and simplicity, we focus on a two-hop system with

\footnotetext{
${ }^{1}$ Our multihop model only considers transmission between adjacent nodes, in contrast to the classical relay channel model where the source and the destination are directly connected [8]. Such a hop-by-hop transmission is a consequence either of physical constraints (e.g., wireline), or of practical design (e.g., treating signals from remote nodes as interference).
} 
a single relay node. The objective of the optimization is to maximize the end-to-end achievable information rate, expressed as the mutual information between the source inputs and the destination outputs, subject to source power allocation and relay processing constraints.

Interestingly, exactly the same rate-maximization problem has been formulated in [11], stemming from the general problem of designing optimal linear source precoder and linear relay transformers for a two-hop system equipped with multiple antennas. Note that through singularvalue decomposition (SVD), multiple-port channel matrices can be decoupled into multicarrier parallel channels. The end-to-end achievable rate, unfortunately, is a non-concave function with power allocation vectors, and thus cannot be readily maximized utilizing techniques like the interior point method or other methods relying on the sufficiency of the Karush-Kuhn-Tucker (KKT) conditions (see, e.g., [9]) in characterizing optimal solutions. Due to this difficulty, no optimal solution was provided in [11]. On the other hand, by extending the proof techniques in an earlier work [10], the authors of [11] established an important structural property of the optimal solution. For the multicarrier system considered here, the structural property implies that the optimal linear relay processing consists of two stages. In the first stage, the incoming subcarriers from the source-relay link should be permuted, such that their rankings with respect to their channel gain magnitudes are matched to the outgoing subcarriers of the relay-destination link. In this way, strong subcarriers are further strengthened, and weak subcarriers are further weakened. This is somewhat analogous to maximal-ratio combining in diversity reception [12]. Subsequently, the second stage corresponds to a collection of per-subcarrier linear AF, and is subject to optimization.

In this paper, we successfully accomplish the optimization of the end-to-end achievable rate, and the corresponding algorithm is a linear search procedure with computational complexity that is linear with the number of subcarriers. Although the optimization problem lacks the desired convexity properties, it is still possible to obtain its solution through an exhaustive enumeration of all of its stationary and boundary points. Generally speaking, the number of those candidate points grows exponentially with the size of the problem (characterized by the number of subcarriers) so that an exhaustive enumeration is practically infeasible. Fortunately, by utilizing the aforementioned structural property regarding optimal permutation as established in [10] and [11], we find that the exhaustive enumeration can be reduced to a linear search, in which, at each step, we compute the maximum rate under the constraint that a certain number of weak subcarriers are not allocated any power. 
The optimal algorithm involves solving a series of cubic equations, which still may not be pragmatic. Further motivated by the structural property and by a simple heuristic, we propose a suboptimal algorithm, in which each hop link simply performs waterfilling separately over a linearly searched subset of strong subcarriers, thus avoiding the occurrence of cubic equations. A surprising observation made empirically via simulations is that the low-complexity, suboptimal algorithm usually achieves near-optimal performance, with negligible rate loss. We note that all the developments in the paper can readily be applicable to a system whose transceivers have multiple input/output ports, as in [10] and [11].

We further present analytical results in the special case of frequency-flat channel profile, i.e., with common channel gain for each subcarrier in a link. For this case, the optimal algorithm and the low-comlexity suboptimal algorithm coincide and the maximum rate is achieved by an on-off power allocation scheme over the subcarriers. This phenomenon precisely reflects the impact of the lack of convexity in the problem. Consequently, even if each hop link is wideband with sufficiently many subcarriers available, it is often optimal not to use all of these subcarriers. In contrast, for single-hop transmission, it is always optimal to uniformly allocate power among all subcarriers in the wideband regime.

The remainder of this paper is organized as follows. Section II introduces the two-hop system model and formulates the rate maximization problem. Section III establishes the linear search algorithm that yields the optimal solution to the rate maximization problem. Section IV motivates and describes the low-complexity suboptimal algorithm. Section V presents results and discussion on the case where the hop links exhibit frequency-flat channel profile. Section VI generalizes the problem to the limit of continuous frequency-domain response. Section VII presents results and discussion from numerical simulation, for subcarriers whose channel coefficients resemble Gaussian random variables. Finally Section VIII concludes the paper. As a convention, all logarithms are to base $e$, and all rates are measured by nats.

\section{System Model AND PROBlem Formulation}

In this paper, we consider a two-hop system, with each hop link being a multicarrier modulated channel consisting of $N$ subcarriers. For simplicity, we assume that the channel responses remain static throughout transmission, and that the inter-carrier interference between subcarriers is negligible. With the time index suppressed, the channel equation for subcarrier $n$ is written 
in discrete-time baseband form as

$$
\mathrm{Y}_{m}[n]=h_{m}[n] \mathrm{X}_{m}[n]+\mathrm{Z}_{m}[n], \quad n=1, \ldots, N,
$$

with $m=1$ denoting the source-relay link and $m=2$ denoting the relay-destination link. For each hop link, the complex-valued inputs $\mathrm{X}_{m}[\cdot]$ have an average power constraint across the $N$ subcarriers,

$$
\mathbf{E}\left[\sum_{n=1}^{N}\left|\mathbf{X}_{m}[n]\right|^{2}\right]=P_{m}, \quad m=1,2 .
$$

The additive noise random variables $Z_{m}[\cdot]$ are modeled as circularly symmetric complex Gaussian, with zero mean and variance $\sigma_{m}^{2}$ for $m=1,2$. We further assume that $Z_{m}[\cdot]$ are mutually independent across time and across subcarriers (i.e., "white" in both time and frequency). The complex-valued channel profile coefficients, $h_{m}[\cdot]$, are modeled as deterministic and known throughout transmission, in light of the static channel assumption.

The relay node that joins the two hop links is the cascade of a permutation stage and an amplification stage, as illustrated in Figure 1. In its operation, the relay first demodulates the time-domain received signal and obtains the signals over the $N$ subcarriers from the sourcerelay link, $\underline{\mathrm{Y}}_{1}=\left(\mathrm{Y}_{1}[1], \ldots, \mathrm{Y}_{1}[N]\right)^{\mathrm{T}}$, accomplished by standard OFDM demodulation, including removing the cyclic prefix, serial-parallel conversion, and discrete Fourier transform (DFT). In the subsequent permutation stage, the relay permutes the elements of the $\underline{Y}_{1}$ vector according to a deterministic permutation function $\pi(\cdot)$, thus obtaining

$$
\underline{\mathrm{Y}}_{1}^{(\pi)}=\left(\mathrm{Y}_{1}[\pi(1)], \ldots, \mathrm{Y}_{1}[\pi(N)]\right)^{\mathrm{T}} \text {. }
$$

Taking $\underline{\mathrm{Y}}_{1}^{(\pi)}$ as its input vector, the amplification stage consists of $N$ linear amplifiers each corresponding to one element in $\underline{Y}_{1}^{(\pi)}$. The output stage vector is thus

$$
\underline{\mathrm{X}}_{2}=\left(c[1] \mathrm{Y}_{1}[\pi(1)], \ldots, c[N] \mathrm{Y}_{1}[\pi(N)]\right)^{\mathrm{T}} .
$$

Note that the amplification coefficients $c[\cdot]$ can be different. The optimal and suboptimal selections of these coefficients will be one of the major contributions of this work. Finally, the relay forms its time-domain transmitted signal for transmission over the relay-destination link, by performing standard OFDM modulation (inverse DFT, adding the cyclic prefix, and parallelto-serial conversion) on $\underline{X}_{2}$. 
The received signal at the destination for subcarrier $n$ is denoted $Y_{2}[n]$; the end-to-end channel equation for $Y_{2}[n]$ is given by,

$$
\begin{aligned}
\mathrm{Y}_{2}[n] & =h_{2}[n] \mathrm{X}_{2}[n]+\mathrm{Z}_{2}[n] \\
& =h_{2}[n] c[n] \mathrm{Y}_{1}\left[\pi^{-1}(n)\right]+\mathrm{Z}_{2}[n] \\
& =h_{2}[n] c[n]\left(h_{1}\left[\pi^{-1}(n)\right] \mathbf{X}_{1}\left[\pi^{-1}(n)\right]+\mathrm{Z}_{1}\left[\pi^{-1}(n)\right]\right)+\mathrm{Z}_{2}[n] \\
& =h_{1}\left[\pi^{-1}(n)\right] h_{2}[n] c[n] \mathrm{X}_{1}\left[\pi^{-1}(n)\right]+\left(h_{2}[n] c[n] \mathbf{Z}_{1}\left[\pi^{-1}(n)\right]+\mathrm{Z}_{2}[n]\right) .
\end{aligned}
$$

To proceed, it is more convenient to rewrite $\mathrm{X}_{m}[n]=\sqrt{\gamma_{m}[n]} \tilde{\mathrm{X}}_{m}[n]$, such that each $\tilde{\mathrm{X}}_{m}[n]$ has zero mean and unit variance, and such that the nonnegative power allocation vector $\underline{\gamma}_{m}=$ $\left(\gamma_{m}[1], \ldots, \gamma_{m}[N]\right)^{\mathrm{T}}$ satisfies the average power constraint $\sum_{n=1}^{N} \gamma_{m}[n]=P_{m}$, for $m=1,2$. With this notation, the amplification coefficients at the relay satisfy

$$
|c[n]|^{2}=\frac{\gamma_{2}[n]}{\left|h_{1}\left[\pi^{-1}(n)\right]\right|^{2} \gamma_{1}\left[\pi^{-1}(n)\right]+\sigma_{1}^{2}}, \quad n=1, \ldots, N .
$$

Consequently, the end-to-end channel equation (5) can be normalized and reduced to

$$
\tilde{\mathrm{Y}}_{2}[n]=\sqrt{\rho[n]} \tilde{\mathrm{X}}_{1}\left[\pi^{-1}(n)\right]+\tilde{\mathrm{Z}}[n], \quad n=1, \ldots, N,
$$

where $\tilde{Z}[n]$ is a circularly symmetric complex Gaussian random variable with zero mean and unit variance, and

$$
\rho[n]=\frac{\left|h_{1}\left[\pi^{-1}(n)\right]\right|^{2}\left|h_{2}[n]\right|^{2} \gamma_{1}\left[\pi^{-1}(n)\right] \gamma_{2}[n]}{\left|h_{1}\left[\pi^{-1}(n)\right]\right|^{2} \gamma_{1}\left[\pi^{-1}(n)\right] \sigma_{2}^{2}+\left|h_{2}[n]\right|^{2} \gamma_{2}[n] \sigma_{1}^{2}+\sigma_{1}^{2} \sigma_{2}^{2}}
$$

characterizes the end-to-end SNR for $\mathrm{Y}_{2}[n]$.

To simplify notation, in the remainder of this paper, we introduce

$$
a_{m}[n]=\frac{\left|h_{m}[n]\right|^{2}}{\sigma_{m}^{2}}, \quad \text { for } m=1,2
$$

as the normalized channel gains, and rewrite the per-subcarrier $\operatorname{SNR} \rho[n]$ as

$$
\rho[n]=\frac{a_{1}\left[\pi^{-1}(n)\right] a_{2}[n] \gamma_{1}\left[\pi^{-1}(n)\right] \gamma_{2}[n]}{a_{1}\left[\pi^{-1}(n)\right] \gamma_{1}\left[\pi^{-1}(n)\right]+a_{2}[n] \gamma_{2}[n]+1} .
$$

For a specified permutation function $\pi(\cdot)$ and power allocation vectors $\underline{\gamma}_{m}, m=1,2$, the theoretically maximum information rate that is achievable over the end-to-end two-hop multicarrier system is

$$
R(\pi(\cdot), \underline{\gamma})=\sum_{n=1}^{N} \log (1+\rho[n])
$$


To achieve the rate $R(\pi(\cdot), \underline{\gamma})$, we assume that all the inputs over the $N$ subcarriers resemble mutually independent and circularly symmetric complex Gaussian random variables, and that optimum encoding/decoding procedures are utilized. Consequently, the problem that we seek to solve in this paper is to maximize the rate, as follows,

$$
\begin{array}{r}
\max R(\pi(\cdot), \underline{\gamma})=\sum_{n=1}^{N} \log (1+\rho[n]), \\
\text { s.t. } \quad \sum_{n=1}^{N} \gamma_{m}[n]=P_{m}, \quad m=1,2, \\
\text { variables: } \quad \pi(\cdot), \underline{\gamma},
\end{array}
$$

where $\rho[n]$ is given by Equation (10).

Remark 1: In the rate function in Equation (12), we implicitly ignore the rate loss due to the cyclic prefix if the multicarrier modulation is implemented by OFDM. Such a loss becomes negligible as we increase the number of subcarriers such that $N$ is much larger than the channel delay spread.

Remark 2: The preceding description of the channel model accommodates both full-duplex and half-duplex relay transceivers. For the half-duplex case, we only need to properly scale the model, namely, double the average power constraint $\left(P_{m}, m=1,2\right)$ and halve the resulting information rate $R(\pi(\cdot), \underline{\gamma})$.

\section{Structure AND Algorithm for Optimal Solution}

The optimization problem (12) can be decoupled into two separate subproblems: a discrete optimization that yields the optimum permutation function $\pi^{*}(\cdot)$, and a continuous optimization that yields the optimum power allocation vectors $\underline{\gamma}_{m}^{*}, m=1,2$. At first glance, optimizing $\pi(\cdot)$ requires an exhaustive search over all possible permutations of $\{1, \ldots, N\}$, whose complexity grows exponentially with $N$ because the total number of possible permutations is $N$ !. Fortunately, by exploiting matrix theory, the authors of [10], [11] were able to circumvent the exhaustive enumeration and prove that the optimal permutation function yields a rather simple form. Furthermore, in this paper, we depart from characterization of optimal solution structures to practical algorithm implementations.

To rephrase the result for our problem in this paper, it is convenient to introduce a ranking operator instead of the permutation function. For a vector, the ranking operator is defined as follows. 
Definition 1: For a length- $N$ vector $\underline{s}=\{s[n]\}_{n=1}^{N}$ whose elements are all nonnegative, the ranking operator $\mathcal{R}$ is defined such that $\mathcal{R}(\underline{s})=\left\{s^{\mathcal{R}}[n]\right\}_{n=1}^{N}$ is another length- $N$ vector, whose elements are obtained by permuting the elements of $\underline{s}$, with $s^{\mathcal{R}}\left[n_{1}\right] \geq s^{\mathcal{R}}\left[n_{2}\right]$ for arbitrary $n_{1}<n_{2}$.

Having defined the ranking operator, we state the optimal permutation function in the following lemma.

Lemma 2: (see [10, Sec. III] and [11, Thm. 1])

For the two-hop transmission system model in Section II, the permutation function $\pi^{*}(\cdot)$ that solves problem (12) is an identity function $\pi(n)=n$ if we apply the ranking operator $\mathcal{R}$ to $\left\{a_{1}[n]\right\}_{n=1}^{N}$ and $\left\{a_{2}[n]\right\}_{n=1}^{N}$ simultaneously.

In words, Lemma 2 states that if we rank and re-index the subcarriers of each hop link according to the rankings of subcarriers' channel gain magnitudes, in a descending order, then no further permutation is needed for achieving the maximum transmission rate. Throughout the remainder of this paper, we shall adhere to this ranking (therefore suppressing the superscript .$^{\mathcal{R}}$ in $\left.\left\{a_{m}^{\mathcal{R}}[n]\right\}_{n=1}^{N}\right), m=1,2$, thus reducing the problem to one of power allocation only. Now it is convenient to rewrite the achievable rate in Equation (12), utilizing Equation (10), as

$$
\begin{aligned}
R(\underline{\gamma}) & =\sum_{n=1}^{N} \log \left(1+a_{1}[n] \gamma_{1}[n]\right)+\sum_{n=1}^{N} \log \left(1+a_{2}[n] \gamma_{2}[n]\right) \\
& -\sum_{n=1}^{N} \log \left(1+a_{1}[n] \gamma_{1}[n]+a_{2}[n] \gamma_{2}[n]\right)
\end{aligned}
$$

where we once again emphasize that the channel profile coefficients have been ranked and reindexed such that $a_{1}[1] \geq a_{1}[2] \geq \ldots \geq a_{1}[N]$ and $a_{2}[1] \geq a_{2}[2] \geq \ldots \geq a_{2}[N]$.

It is easy to verify that $R(\underline{\gamma})$ is not a concave function, and thus its maximization cannot be solved by techniques like the interior point method or other methods relying on the sufficiency of the Karush-Kuhn-Tucker (KKT) conditions in characterizing optimal solutions. Actually, the KKT conditions can only yield all possible candidates of the optimal solution, whereas enumerating all those candidate solutions is still overwhelming in complexity. What can be said is that, in the optimal solution, for each subcarrier index $n \in\{1, \ldots, N\}$, we have either

$$
\begin{array}{ll} 
& \frac{\partial J(\underline{\gamma})}{\partial \gamma_{1}[n]}=\frac{\partial J(\underline{\gamma})}{\partial \gamma_{2}[n]}=0 ; \\
\text { or, } \quad & \gamma_{1}[n]=\gamma_{2}[n]=0
\end{array}
$$


where the Lagrangian $J(\underline{\gamma})$ is

$$
J(\underline{\gamma})=R(\underline{\gamma})-\lambda_{1}\left(\sum_{n=1}^{N} \gamma_{1}[n]-P_{1}\right)-\lambda_{2}\left(\sum_{n=1}^{N} \gamma_{2}[n]-P_{2}\right),
$$

for arbitrary $\lambda_{m} \in \mathbb{R}, m=1,2$. The vanishing partial derivatives in Equation (16), are from the first-order optimality test. In Equation (17), the variables are on the boundary of the feasible set, and we note that if either of $\gamma_{m}[n], m=1,2$, vanishes, the other also has to be so in the optimal solution.

Applying Lemma 2, we obtain that the optimal power allocation solution exhibits the following truncated structure.

Lemma 3: For the optimal power allocation vectors $\underline{\gamma}_{m}^{*}, m=1,2$, there exists an integer $K \in\{1, \ldots, N\}$, such that

$$
\begin{aligned}
\gamma_{m}^{*}[n] & >0, \quad \forall n \leq K \\
\text { and } \gamma_{m}^{*}[n] & =0, \quad \text { otherwise }
\end{aligned}
$$

for $m=1,2$.

Proof: This is a direct consequence of the ranking with $a_{1}[1] \geq a_{1}[2] \geq \ldots \geq a_{1}[N]$ and $a_{2}[1] \geq a_{2}[2] \geq \ldots \geq a_{2}[N]$. Actually, assume that there exist $n_{1}$ and $n_{2}, n_{1}<n_{2}$, with at least one of the inequalities $a_{m}\left[n_{1}\right] \geq a_{m}\left[n_{2}\right], m=1,2$, being strict, and with $\gamma_{m}^{*}\left[n_{1}\right]=0$ and $\gamma_{m}^{*}\left[n_{2}\right]>0, m=1,2$, in the optimal solution. Then if we exchange the values of $\gamma_{m}^{*}\left[n_{1}\right]$ and $\gamma_{m}^{*}\left[n_{2}\right]$, the resulting achievable rate will always be increased because we have $a_{m}\left[n_{1}\right] \geq a_{m}\left[n_{2}\right]$, $m=1,2$, with at least one of them being strict, and because $R(\gamma)$ is a monotonically increasing function with each $\gamma_{m}[\cdot] \geq 0, m=1,2$.

The implication of Lemma 3 is that we can adopt a linear search procedure to seek the optimal power allocation vectors and the maximum achievable rate. Due to the truncated structure of the optimal solution, all the power allocation variables satisfying the boundary condition (17) are grouped together around the weak subcarriers, and all the remaining power allocation variables have to be positive and satisfy the first-order optimality condition (16). In the $k$-th step of the linear search, we explicitly enforce $\gamma_{m}[n]=0, m=1,2$, for all $n>k$, solve $\gamma_{m}[n], m=1,2$, for $n \leq k$ according to Equation (16), and simultaneously find $\lambda_{1}, \lambda_{2}$ such that the resulting power constraints (13) are satisfied. For each $k \in\{1, \ldots, N\}$ the above procedure yields a pair of power allocation vectors and a resulting achievable rate, and the solution to the optimization 
problem (12) corresponds to the maximum among the obtained $N$ rates. Such a procedure has a complexity that is linear in the number of subcarriers.

Formally, we can describe the linear search procedure in details, as follows.

Proposition 1: For the rate maximization problem (12), the optimal solution can be obtained through the following linear search algorithm.

\section{The optimal linear search algorithm}

Initialization Set $k=1, \gamma_{1}[1]=P_{1}, \gamma_{2}[1]=P_{2}$, and $\gamma_{m}[n]=0, m=1,2$, for all $n>1$. Compute $\tilde{R}_{1}=R(\underline{\gamma})$ according to Equation (15).

Execution For $k$ from 2 to $N$ :

(a) Set $\gamma_{m}[n]=0, m=1,2$, for all $n>k$ (if $k=N$, skip).

(b) Find $\lambda_{m}>0, m=1,2$, such that the positive solutions $\left\{\gamma_{m}[n]\right\}_{n=1}^{k}, m=1,2$, of the following equations:

$$
\begin{aligned}
& \frac{a_{1}[n]}{1+a_{1}[n] \gamma_{1}[n]}-\frac{a_{1}[n]}{1+a_{1}[n] \gamma_{1}[n]+a_{2}[n] \gamma_{2}[n]}=\lambda_{1} \\
& \frac{a_{2}[n]}{1+a_{2}[n] \gamma_{2}[n]}-\frac{a_{2}[n]}{1+a_{1}[n] \gamma_{1}[n]+a_{2}[n] \gamma_{2}[n]}=\lambda_{2},
\end{aligned}
$$

for $n=1, \ldots, k$, satisfy the power constraints

$$
\sum_{n=1}^{k} \gamma_{m}[n]=P_{m}, \quad m=1,2
$$

Remark 1: If for certain $n$, multiple positive solutions exist for Equations $(21,22)$, compare the resulting values of the corresponding term in $J(\underline{\gamma})$, and select the one that leads to the maximum; c.f., e.g., [13].

Remark 2: If for certain $n$, no positive solutions exist for Equations $(21,22)$, then the corresponding pair of $\lambda_{m}, m=1,2$, does not satisfy the power constraints and therefore should be discarded.

(c) Compute $\tilde{R}_{k}=R(\underline{\gamma})$ according to Equation (15) using the power allocation vectors obtained in (b).

Termination Choose $k \in\{1, \ldots, N\}$ that achieves the maximum $\tilde{R}_{k}$, which then is the outcome of the optimization problem (12), and the associated power allocation vectors are the optimal solution. 
In the linear search algorithm described, the key part is step (b) in the execution, where for each $1 \leq n \leq k$, a pair of equations $(21,22)$ need be solved. For implementation, these two equations can be solved through a cubic equation as follows,

$$
\begin{aligned}
& \text { Let } t=\frac{1}{1+a_{1}[n] \gamma_{1}[n]}, \text { where } t \text { is the positive solution of } \\
& t^{3}-\left(1+\frac{2 \lambda_{1}}{a_{1}[n]}-\frac{\lambda_{2}}{a_{2}[n]}\right) t^{2}+\frac{\lambda_{1}}{a_{1}[n]}\left(2+\frac{\lambda_{1}}{a_{1}[n]}-\frac{\lambda_{2}}{a_{2}[n]}\right) t-\frac{\lambda_{1}}{a_{1}[n]}\left(\frac{\lambda_{1}}{a_{1}[n]}-\frac{\lambda_{2}}{a_{2}[n]}\right)=0 ; \\
& \text { then } \quad \gamma_{1}[n]=\frac{1}{a_{1}[n]}\left(\frac{1}{t}-1\right), \\
& \quad \gamma_{2}[n]=\frac{1}{a_{2}[n]}\left[\left(t-\frac{\lambda_{1}}{a_{1}[n]}+\frac{\lambda_{2}}{a_{2}[n]}\right)^{-1}-1\right] .
\end{aligned}
$$

We note that, finding $\lambda_{m}>0, m=1,2$, that satisfy the power contraints involves a twodimensional grid search, which can be implemented in principle through discretization, because from Equations $(21,22)$ it is obvious that $0<\lambda_{m}<\min _{n \leq k} a_{m}[n], m=1,2$, corresponding to a bounded area.

\section{Low-Complexity Suboptimal Algorithm BASED ON WATERfilling}

In this section, we propose a low-complexity algorithm which for the general case is usually near-optimal and is optimal for a specific set of channel conditions. The idea is a simple heuristic stemming from Equation (15); that is, in Equation (15) we only maximize the first two summations (easily accomplished through waterfilling the two hop links separately) without considering the loss in the third. Meanwhile, in light of the structure of the optimal solution developed in Lemma 3, we again adopt a linear search procedure, rather than always activating all the $N$ subcarriers.

The low-complexity algorithm is described in details as follows.

\section{A low-complexity linear search algorithm}

Initialization Set $k=1, \gamma_{1}[1]=P_{1}, \gamma_{2}[1]=P_{2}$, and $\gamma_{m}[n]=0, m=1,2$, for all $n>1$. Compute $\tilde{R}_{1}=R(\underline{\gamma})$ according to Equation (15).

Execution For $k$ from 2 to $N$ :

(a) Set $\gamma_{m}[n]=0, m=1,2$, for all $n>k$ (if $k=N$, skip).

(b) Find $\lambda_{m}>0, m=1,2$, with

$$
\gamma_{m}[n]=\max \left(\frac{1}{\lambda_{m}}-\frac{1}{a_{m}[n]}, 0\right), \quad m=1,2, \text { and } n=1, \ldots, k,
$$


to satisfy the power constraints

$$
\sum_{n=1}^{k} \gamma_{m}[n]=P_{m}, \quad m=1,2
$$

(c) Compute $\tilde{R}_{k}=R(\underline{\gamma})$ according to Equation (15) using the power allocation vectors obtained in (b).

Termination Choose $k \in\{1, \ldots, N\}$ that achieves the maximum $\tilde{R}_{k}$, which then is the outcome of the algorithm.

It is clear that step (b), here, generally leads to a power allocation different from that of the optimal algorithm in Section III. However, the attendant computational complexity is substantially reduced, hence rendering the algorithm attractive for practical purposes.

For the above low-complexity algorithm, we can establish a result regarding the permutation function, as given by the following proposition.

Proposition 2: In the low-complexity linear search algorithm described above, even if we are allowed to choose any permutation function to re-index the subcarriers, the optimal permutation function is still the same as that given by Lemma 2 .

Proof: We shall prove that for any value of $k$ in the algorithm execution, the optimal re-indexing of the subcarriers should be such that $a_{1}[1] \geq a_{1}[2] \geq \ldots \geq a_{1}[N]$ and $a_{2}[1] \geq a_{2}[2] \geq \ldots \geq$ $a_{2}[N]$. From the waterfilling step in the algorithm, we observe that if we adopt the re-indexing implied by Lemma 2 , the overall signal strengths also satisfy $a_{1}[1] \gamma_{1}[1] \geq a_{1}[2] \gamma_{1}[2] \geq \ldots \geq$ $a_{1}[N] \gamma_{1}[N]$, and $a_{2}[1] \gamma_{2}[1] \geq a_{2}[2] \gamma_{2}[2] \geq \ldots \geq a_{2}[N] \gamma_{2}[N]$. Hence now it suffices to prove the following inequality: for any two length- $N$ nonnegative real vectors $\left\{u_{n}\right\}_{n=1}^{N}$ and $\left\{v_{n}\right\}_{n=1}^{N}$, both sorted in descending order, we have

$$
\sum_{n=1}^{N} \log \left(1+u_{n}+v_{n}\right) \leq \sum_{n=1}^{N} \log \left(1+u_{\pi(n)}+v_{n}\right)
$$

for any permutation function $\pi(\cdot)$. By invoking certain matrix inequalities, (26) can be shown to be a special case of the development in [10]. Herein we provide an alternative direct proof. Since any permutation function can be decomposed into the concatenation of a series of switching operations, each exchanging the indexes of two elements, ${ }^{2}$ it suffices to show that each such

\footnotetext{
${ }^{2}$ For example, consider $\left(u_{1}, u_{2}, u_{3}, u_{4}\right)$ in descending order, then $\left(u_{3}, u_{1}, u_{4}, u_{2}\right)$ can be obtained through the following three switching operations in sequential order: $u_{1} \leftrightarrow u_{2}$ (obtaining $\left(u_{2}, u_{1}, u_{3}, u_{4}\right)$ ), $u_{2} \leftrightarrow u_{3}$ (obtaining $\left(u_{3}, u_{1}, u_{2}, u_{4}\right)$ ), and finally $u_{2} \leftrightarrow u_{4}$.
} 
switching operation does not decrease the value of the left hand side of the inequality (26). For this, suppose we exchange $u_{k}$ and $u_{l}$ with $l<k$ (hence $u_{l} \geq u_{k}$ and $v_{l} \geq v_{k}$ ). Then the net increment due to this switching operation is

$$
\begin{aligned}
& \log \left(1+u_{l}+v_{k}\right)+\log \left(1+u_{k}+v_{l}\right)-\log \left(1+u_{l}+v_{l}\right)-\log \left(1+u_{k}+v_{k}\right) \\
= & \log \frac{1+u_{k}+v_{k}+u_{l}+v_{l}+u_{k} u_{l}+v_{k} v_{l}+u_{k} v_{k}+u_{l} v_{l}}{1+u_{k}+v_{k}+u_{l}+v_{l}+u_{k} u_{l}+v_{k} v_{l}+u_{k} v_{l}+u_{l} v_{k}} \\
\geq & 0,
\end{aligned}
$$

since $u_{k} v_{k}+u_{l} v_{l}-u_{k} v_{l}-u_{l} v_{k}=\left(u_{l}-u_{k}\right)\left(v_{l}-v_{k}\right) \geq 0$. This finalizes the proof of Proposition 2.

\section{Hops With Frequency-Flat Channel Profile}

If the two hop links in the system model both have a frequency-flat channel profile, i.e., $a_{1}[n]=g_{1}$ and $a_{2}[n]=g_{2}$ for all $n=1, \ldots, N$, then from the linear search optimization algorithm in Section III we readily find that the optimal power allocation vectors take an on-off form, and that the maximum achievable rate can be written as

$$
R^{*}=\max _{K \in\{1, \ldots, N\}} K \cdot \log \left[\frac{\left(1+g_{1} P_{1} / K\right)\left(1+g_{2} P_{2} / K\right)}{1+\left(g_{1} P_{1}+g_{2} P_{2}\right) / K}\right] .
$$

If we denote the maximizer $K$ by $K^{*}$, then the optimal power allocation is to arbitrarily let $K$ out of the $N$ subcarriers be endowed with power $P_{m} / K, m=1,2$, and to not use the remaining $(N-$ $K)$ subcarriers, for each hop link. Also, there is no need to use any specific permutation function, besides enforcing that no "on" subcarrier is connected to any "off" subcarriers. Intuitively, the simplicity of the solution in the frequency-flat channel profile case is because all the subcarriers are homogeneous.

From Equation (28), the maximization problem can be approximated by the following relaxed form:

$$
R^{*} \approx \max _{t \in[1 / N, 1]} \frac{1}{t} \cdot \log \left[\frac{\left(1+g_{1} P_{1} t\right)\left(1+g_{2} P_{2} t\right)}{1+\left(g_{1} P_{1}+g_{2} P_{2}\right) t}\right] .
$$

Here we note that the real-valued variable $t$ is no longer restricted to be the reciprocal of integers. For the underlying function

$$
f(t)=\frac{1}{t} \cdot \log \left[\frac{\left(1+g_{1} P_{1} t\right)\left(1+g_{2} P_{2} t\right)}{1+\left(g_{1} P_{1}+g_{2} P_{2}\right) t}\right],
$$


over $t \in(0,1]$ in Equation (29), it can be shown that it has a unique maximum and asymptotically vanishes as $t \rightarrow 0$, for any finite $\left(g_{m}, P_{m}\right), m=1,2$. Denote the maximizer $t$ by $t^{*}$. It then follows that

$$
\begin{aligned}
\text { If } N \geq 1 / t^{*}, & K^{*}=\left\lfloor 1 / t^{*}\right\rfloor \text { or }\left\lceil 1 / t^{*}\right\rceil ; \\
\text { else, } & K^{*}=N .
\end{aligned}
$$

That is, when the number of subcarriers $N$ is relatively small, it is optimal to allocate power uniformly over all of them; however, when $N$ exceeds a threshold, there is no further benefit from spreading power, and instead the optimal number of subcarriers remains fixed. In fact, since $\lim _{t \rightarrow 0^{+}} f(t)=0$, uniform wideband power allocation only leads to vanishing achievable rates.

To illustrate the analysis, we plot in Figures 2 and 3 the bahavior of $1 / t^{*}$ and $R^{*}$, for the case where $g_{1} P_{1}=g_{2} P_{2}$, i.e., both hop links have identical SNR. In Figure 2 it is evident that the optimal number of subcarriers $\left(K^{*} \approx 1 / t^{*}\right)$ rapidly grows with $\mathrm{SNR}$, approximately at a linear speed in the log-log plot. Consequently, in Figure 3 we notice that the maximum achievable rate $\left(R^{*}\right)$ rapidly increases with hop links' SNR.

Not immediately evident, but a closer look at Figure 3 reveals that the ratio between $R^{*}$ and $g_{m} P_{m}, m=1,2$, is actually a constant approximately equal to 0.3 . Note that therein $g_{m} P_{m}, m=$ 1,2 , can be shown to be nothing but the two-hop channel capacity using the DF scheme in the wideband limit. So this observation shows that the optimal frequency-domain non-regenerative relay processing can achieve an information rate linearly growing with SNR but with a reduced growth slope. To further elaborate on this issue, let $g_{1} P_{1}=P$ and $g_{2} P_{2}=\beta P$, where $\beta>0$ is fixed as $P$ changes. Now consider the performance metric

$$
\frac{f(t)}{P}=\frac{1}{P t} \cdot \log \left[\frac{(1+P t)(1+\beta P t)}{1+(1+\beta) P t}\right],
$$

which is the ratio between the achievable rate upon activating $1 / t$ subcarriers and $P$, and can be maximized by changing $P t$ (equivalent to changing $K / P$, the SNR-normalized number of active subcarriers), for any given $\beta$. In Figure 4 we plot the relationship between $K^{*} / P$ and $\beta$ (in $\mathrm{dB}$ ). As can be seen, the optimal number of active subcarriers increases with $\beta$; for example, if $\beta=1$ $(0 \mathrm{~dB})$, then it is optimal to activate approximately $0.7 P$ subcarriers. In Figure 5 we further plot the relationship between $R^{*} / P$ and $\beta$ (in $\mathrm{dB}$ ). To verify our observation in Figure 3 , we can see that $R^{*} / P \approx 0.3$ at $\beta=1$. As $\beta$ increases (i.e., the relay-destination hop link becomes less 
noisy), $R^{*} / P$ gradually approaches one, corresponding to the wideband DF capacity $P$. This is because as $\beta \rightarrow \infty$, the relay-destination link effectively vanishes, and the two-hop system is reduced to a single-hop system with the source-relay link only.

\section{Vi. Continuous Frequency-Domain Channel Response}

In the previous sections, we focus on multicarrier channels with a fixed number of subcarriers. In physical systems, a multicarrier channel is obtained from discretizing a continuous frequencydomain channel response into a collection of discrete frequency bins over each of which the frequency response is approximately "flat". In this section, we therefore present the problem in the limit of continuous frequency-domain channel responses, and analyze the behavior of the low-complexity suboptimal algorithm in Section IV.

We start with a two-hop network in which each hop link is described by a continuous-time real baseband channel [14], as

$$
\mathrm{Y}_{m}^{t}(t)=h_{m}^{t}(t) * \mathrm{X}_{m}^{t}(t)+\mathrm{Z}_{m}^{t}(t)
$$

where the superscript ${ }^{t}$ indicates that the variables are in time, and the subscript ${ }_{m}=1,2$ denotes the source-relay link or the relay-destination link, respectively. The additive noise process $Z_{m}^{t}(t)$ is white Gaussian, with one-sided power spectral density $N_{0}$. The channel input process $X_{m}^{t}(t)$ is linearly convolved with the channel impulse response $h_{m}^{t}(t)$, modeling a linear-filter channel. As we take Fourier transform of Equation (32), we obtain the frequency-domain description of the hop links

$$
\mathrm{Y}_{m}(f)=h_{m}(f) \mathrm{X}_{m}(f)+\mathrm{Z}_{m}(f)
$$

The continuous frequency-domain channel response $h_{m}(f)$ is the Fourier transform of $h_{m}^{t}(t)$.

For the subsequent exposition, it is convenient to extend the ranking operator from vectors to continuous functions, as given in the following definition.

Definition 4: For a measurable nonnegative real function $g(f)$ over the positive real line $f \geq 0$, the ranking operator $\mathcal{R}$ is defined such that $\mathcal{R} \circ g(f)$ is another measurable nonnegative real function over the positive real line, denoted by $g^{\mathcal{R}}(f)$, satisfying:

1) For each $\alpha \geq 0$, we have

$$
\mu(\{f: g(f) \leq \alpha\})=\mu\left(\left\{f: g^{\mathcal{R}}(f) \leq \alpha\right\}\right),
$$

where $\mu(\cdot)$ denotes the Lebesgue measure on the real line. 
2) For arbitrary $0 \leq f_{1}<f_{2}$, we have

$$
g^{\mathcal{R}}\left(f_{1}\right) \geq g^{\mathcal{R}}\left(f_{2}\right) .
$$

Intuitively, the ranking operator is used to "re-index" the frequencies such that the resulting frequency-domain channel responses are monotonically decreasing with frequency. Consequently, no further permutation is needed in any discretized multicarrier systems. For simplicity, we assume that for each $m=1,2$, both $a_{m}(f)=\left|h_{m}(f)\right|^{2} / N_{0}$ and $a_{m}^{\mathcal{R}}(f)$ are sufficiently smooth with piecewise continuous derivatives.

Now as we discretize the frequency line into infinitesimal bins and perform multicarrier modulation over them, the resulting rate maximization problem for frequency-domain nonregenerative relaying can be formulated in the following form:

$$
\begin{aligned}
\max \quad & \int_{0}^{\infty} \log (1+\rho(f)) d f, \\
& \text { s.t. } \quad \int_{0}^{\infty} \gamma_{m}(f) d f=P_{m}, \quad m=1,2,
\end{aligned}
$$

where the SNR density function $\rho(f)$ is given by

$$
\rho(f)=\frac{a_{1}^{\mathcal{R}}(f) a_{2}^{\mathcal{R}}(f) \gamma_{1}(f) \gamma_{2}(f)}{a_{1}^{\mathcal{R}}(f) \gamma_{1}(f)+a_{2}^{\mathcal{R}}(f) \gamma_{2}(f)+1} .
$$

With sufficiently fine discretization, it is in principle possible to carry out the linear search procedure in Section III to find out the optimal solution to Equation (36). In practice, however, the computational complexity of such a procedure is expected to be still prohibitive. Here we turn to analyzing the behavior of the low-complexity suboptimal algorithm as proposed in Section IV, for which we can establish analytical results.

Following Section IV, for the ranked frequency-domain channel responses $a_{m}^{\mathcal{R}}(f), m=1,2$, we need to find out a cutoff frequency $\bar{f}>0$, such that the transmitted signals have strictly positive power density below $\bar{f}$, and do not contain any component beyond $\bar{f}$. There exists a maximum value for possible $\bar{f}$, since if $\bar{f}$ is chosen too large, the waterfilling procedure would enforce some frequency components below $\bar{f}$ to be zero. To determine the maximum possible $\bar{f}$, we note that for a valid $\bar{f}$, we have

$$
\gamma_{m}(f)=\frac{1}{\lambda_{m}}-\frac{1}{a_{m}^{\mathcal{R}}(f)}, \quad m=1,2, \text { and } \forall 0 \leq f \leq \bar{f},
$$

and

$$
\int_{0}^{\bar{f}} \gamma_{m}(f) d f=P_{m}, \quad m=1,2 .
$$


Hence $\lambda_{m}, m=1,2$, should satisfy

$$
\frac{1}{\lambda_{m}}=\frac{1}{\bar{f}} \int_{0}^{\bar{f}} \frac{1}{a_{m}^{\mathcal{R}}(f)} d f+\frac{P_{m}}{\bar{f}} .
$$

On the other hand, for each $m=1,2$, since $a_{m}^{\mathcal{R}}(f)$ is monotonically decreasing with $f$, we need to have $1 / \lambda_{m} \geq 1 / a_{m}^{\mathcal{R}}(\bar{f})$ to ensure $\gamma_{m}(f)>0$ for all $f<\bar{f}$. Therefore the maximum possible $\bar{f}$ is the lesser between the solutions of the two equations

$$
\frac{1}{\bar{f}} \int_{0}^{\bar{f}} \frac{1}{a_{m}^{\mathcal{R}}(f)} d f+\frac{P_{m}}{\bar{f}}=\frac{1}{a_{m}^{\mathcal{R}}(\bar{f})}, \quad m=1,2 .
$$

Let us denote the maximum possible $\bar{f}$ by $\bar{f}_{\text {max }}$. For each cutoff frequency $\bar{f} \leq \bar{f}_{\text {max }}$, the achievable rate is

$$
R(\bar{f})=\int_{0}^{\bar{f}}\left[\log \left(\frac{a_{1}^{\mathcal{R}}(f)}{\lambda_{1}}-1\right)+\log \left(\frac{a_{2}^{\mathcal{R}}(f)}{\lambda_{2}}-1\right)-\log \left(\frac{a_{1}^{\mathcal{R}}(f)}{\lambda_{1}}+\frac{a_{2}^{\mathcal{R}}(f)}{\lambda_{2}}-1\right)\right] d f,
$$

where $\lambda_{m}, m=1,2$, are given by Equation (41). The maximum rate of the low-complexity suboptimal algorithm is therefore $\max _{0 \leq \bar{f} \leq \bar{f}_{\max }} R(\bar{f})$.

\section{Simulation Results}

In this section, we study the performance of the algorithms presented in Sections III and IV, via Monte Carlo simulations as a theoretical analysis appears elusive (except for certain special cases as in Section V).

For each realization of the two-hop $N$-subcarrier system, we randomly generate the link channel profile coefficients $\left\{a_{m}[n]\right\}_{n=1}^{N}, m=1,2$. Specifically, these coefficients are generated as the squared magnitudes of $2 N$ independent and identically distributed (i.i.d.) circularly symmetric complex Gaussian random variables with zero mean and unit variance. Therefore the $\left\{a_{m}[n]\right\}_{n=1}^{N}$, $m=1,2$, are a collection of i.i.d. exponentially distributed random variables, corresponding to the standard Rayleigh fading model. Such a link model may be a suitable choice for wireless transmission over rich-scattering propagation environments with abundant multipath and without significant line-of-sight paths. Simulation results for other transmission systems can be similarly generated and are not presented in this paper.

In simulation we compute the following end-to-end achievable rates for comparison.

1) The capacity of the two-hop transmission system, which is achieved by the DF scheme, with the source and the relay performing waterfilling to optimally allocate power among subcarriers. The resulting rates will be indicated by "DF" in plots. 
2) The rate achieved by the conventional $\mathrm{AF}$ scheme, in which the power is uniformly allocated among subcarriers for both the source-relay link and the relay-destination link. The resulting rates will be indicated by "AF" in plots.

3) The rate achieved by the optimal algorithm in Section III. The resulting rates will be indicated by "optimal algorithm" in plots.

4) The rate achieved by the low-complexity suboptimal algorithm in Section IV. The resulting rates will be indicated by "suboptimal algorithm" in plots.

To get a qualitative view of the execution of the algorithms, we plot in Figure 6 the snapshot corresponding to a particular channel realization sample with $N=16$ and $P_{1}=P_{2}=20 \mathrm{~dB}$. The $\mathrm{x}$-axis indicates the number of active subcarriers through the execution of algorithm, and the y-axis correspondingly indicates the achieved rate $\tilde{R}_{k}$ when the $k$ strongest subcarriers are activated. Comparing the curves for the optimal (curve with circles) and the suboptimal (curve with squares) algorithms, we find that the performance of the two algorithms are fairly close. Both algorithms show that the maximum rates are achieved when the number of active subcarriers is ten, implying that it is not optimal to activate all the available subcarriers. In particular, for the suboptimal algorithm, it is seen that the achievable rate actually decreases as $k$ goes beyond ten.

For comparison, the capacity (achieved by DF) and the AF achievable rate are indicated in Figure 6 as well. Even for the relatively small number of subcarriers $(N=16)$, we observe that the multicarrier relay processing does not lead to a large performance gain compared with the conventional scalar AF scheme.

To futher quantify the small gap between the optimal algorithm and the low-complexity suboptimal algorithm, we plot in Figure 7 the empirical histogram of the ratio between the rates achieved by the two algorithms. Due to the slow execution of the optimal algorithm in practice, we take $N=16$, a relatively small number. The number of channel realizations is 300 in generating Figure 7. Surprisingly, we notice that most of the time the gap is less than two percent within the optimal performance. This observation is quite noteworthy, especially given the simplicity of the suboptimal algorithm.

We plot in Figure 8 the empirical cumulative distribution functions (CDF) of the achievable rates, for a system with parameters of $N=16$ and $P_{1}=P_{2}=20 \mathrm{~dB}$. The number of channel realizations is 300 . Obviously the CDF of the capacity (achieved by DF) dominates those of the other transmission strategies, while the CDF of the AF achievable rate is the most inferior. The 
CDF of the optimal algorithm and the suboptimal algorithm virtually overlap with each other without much noticeable difference, and lie between that of the capacity and that of the AF achievable rate.

The benefit from the multicarrier relay processing over the conventional scalar AF becomes more significant as the number of subcarriers grows large, and this is illustrated by the empirical CDF in Figure 9, for $N=256$ and $P_{1}=P_{2}=20 \mathrm{~dB}$. We do not present the CDF of the optimal algorithm due to its slow execution for large $N$. Even for the suboptimal algorithm, we notice that, on average, it yields almost a three-fold increase over the AF scheme.

The surprisingly effectiveness of the low-complexity suboptimal algorithm provides us with useful engineering insights, and perhaps is worth further attention for other similar design problems.

\section{CONCLUSIONS}

As the paradigm of communication shifts from point-to-point links to connected networks, there arise numerous novel signal processing problems in designing and analyzing efficient communication strategies. In this paper, realizing the inherent limitations of conventional scalar AF relaying schemes, we formulate the problem of frequency-domain non-regenerative relay processing, for information transmission over multicarrier two-hop systems. The novelty of our discovery is that, even though the problem of jointly optimizing source and relay power allocation is not amenable to standard convex optimization techniques due to its lack of convexity, the optimal solution exhibits a convenient truncated structure and thus can be obtained through a search procedure whose complexity grows linearly rather than exponentially with the number of subcarriers. Furthermore, combining the truncated structure and a simple heuristic, we obtain a low-complexity suboptimal algorithm in which the optimization of the source and the relay is largely separated as individual waterfilling.

The implementation of the proposed algorithms demands knowledge of the channel profile coefficients at the source, the relay, and the destination, thus rendering it in a certain sense less attractive for mobile wireless systems where the channel may change with time rapidly. However, for quasi-static wireless systems like mesh networks or indoor local-area networks, and for wireline systems like DSL, the frequency-domain non-regenerative relaying algorithms may be a possible solution, especially if low-complexity or low-latency relay processing is needed. Future research problems include investigating the algorithms' performance under imperfect 
channel knowledge, extending the problem and its algorithms to multihop transmission systems with more than two hops, and combining the problem with interference mitigation in DSL systems.

\section{ACKNOWLEDGMENT}

The authors wish to thank Prof. Yingbo Hua of University of California Riverside and Mr. Xiaojun Tang of Rutgers University for helpful discussion.

\section{REFERENCES}

[1] W. Zhang and U. Mitra, "Channel-Adaptive Frequency-Domain Relay Processing in Multicarrier Multihop Transmission," in Proc. IEEE International Conference on Acoustic, Speech, and Signal Processing (ICASSP), Las Vegas, NV, Mar.-Apr. 2008.

[2] M. Haenggi and D. Puccinelli, "Routing in Ad Hoc Networks: A Case for Long Hops," IEEE Communication Magazine, Vol. 43, No. 10, pp. 93-101, Oct. 2005.

[3] M. Sikora, J. N. Laneman, M. Haenggi, D. J. Costello, Jr., and T. E. Fuja, "Bandwidth- and Power-Efficient Routing in Linear Wireless Networks,” IEEE Trans. Inform. Theory, Vol. 52, No. 6, pp. 2624-2633, Jun. 2006.

[4] U. Niesen, C. Fragouli, and D. Tuninetti, "Scaling Laws for Line Networks: From Zero-Error to Min-Cut Capacity," in Proc. IEEE International Symposium on Information Theory (ISIT), Seattle, WA, Jul. 2006.

[5] Ö. Oyman and S. Sandhu, "Non-Ergodic Power-Bandwidth Tradeoff in Linear Multi-hop Networks," in Proc. IEEE International Symposium on Information Theory (ISIT), Seattle, WA, Jul. 2006.

[6] W. Zhang and U. Mitra, "Multihopping Strategies: An Error-Exponent Comparison," in Proc. IEEE International Symposium on Information Theory (ISIT), Nice, France, Jun. 2007.

[7] E. C. Posner and A. L. Rubin, "The Capacity of Digital Links in Tandem,” IEEE Trans. Inform. Theory, Vol. 30, No. 3, pp. 464-470, May 1984.

[8] T. M. Cover and J. A. Thomas, Elements of Information Theory, John Wiley \& Sons, Inc., New York, 1991.

[9] S. Boyd and L. Vandenberghe, Convex Optimization, Cambridge University Press, 2004.

[10] X. Tang and Y. Hua, "Optimal Design of Non-Regenerative MIMO Wireless Relays," IEEE Trans. Wireless Commun., Vol. 6, No. 4, pp. 1398-1407, Apr. 2007.

[11] Z. Fang, Y. Hua, and J. C. Koshy, “Joint Source and Relay Optimization for a Non-Regenerative MIMO Relay," in Proc. IEEE Workshop on Sensor Array and Multi-channel Processing, Waltham, MA, Jul. 2006.

[12] D. G. Brennan, "Linear Diversity Combining Techniques," Proc. IRE, Vol. 47, No. 1, pp. 1075-1102, Jun. 1959.

[13] R. Cendrillon, J. Huang, M. Chiang, and M. Moonen, "Autonomous Spectrum Balancing for Digital Subscriber Lines," IEEE Trans. Signal Process., Vol. 55, No. 8, pp. 4241-4257, Aug. 2007.

[14] G. D. Forney, Jr. and G. Ungerboeck, "Modulation and Coding for Linear Gaussian Channels," IEEE Trans. Inform. Theory, Vol. 44, No. 6, pp. 2384-2415, Oct. 1998.

\section{[Authors' Bio]}




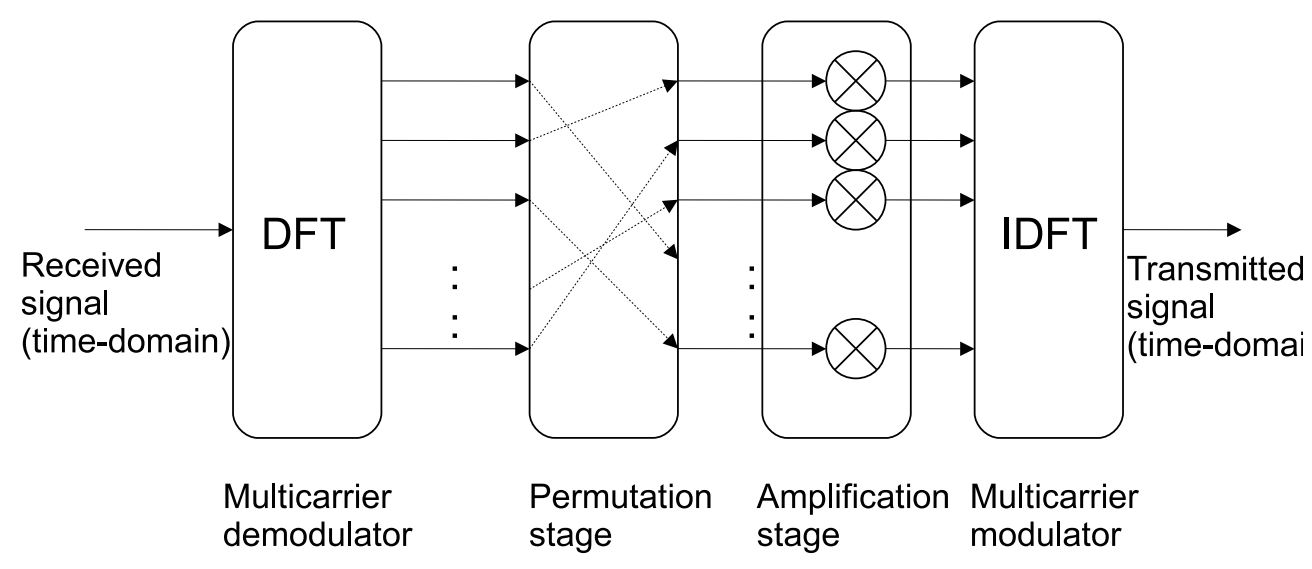

Fig. 1. Schematic illustration of the relay processing. Note that the additional standard multicarrier modulation/demodulation steps, viz., adding/removing cyclic prefix and parallel/serial conversions are not explicitly displayed.

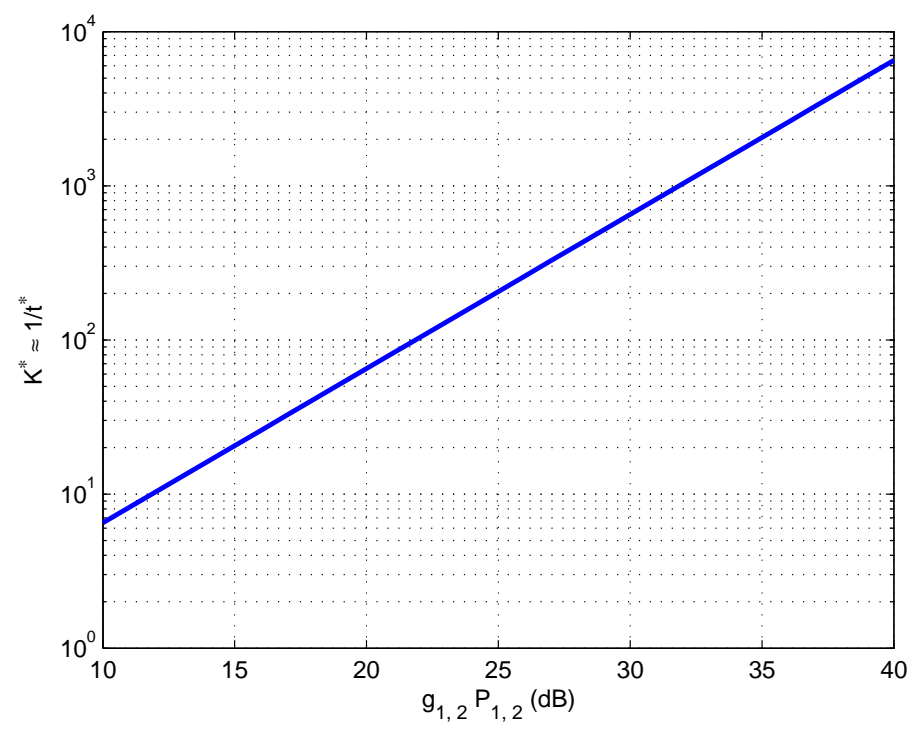

Fig. 2. The optimal number of subcarriers in wideband systems, as a function of hop links' SNR (both hop links have identical SNR in the plot). 


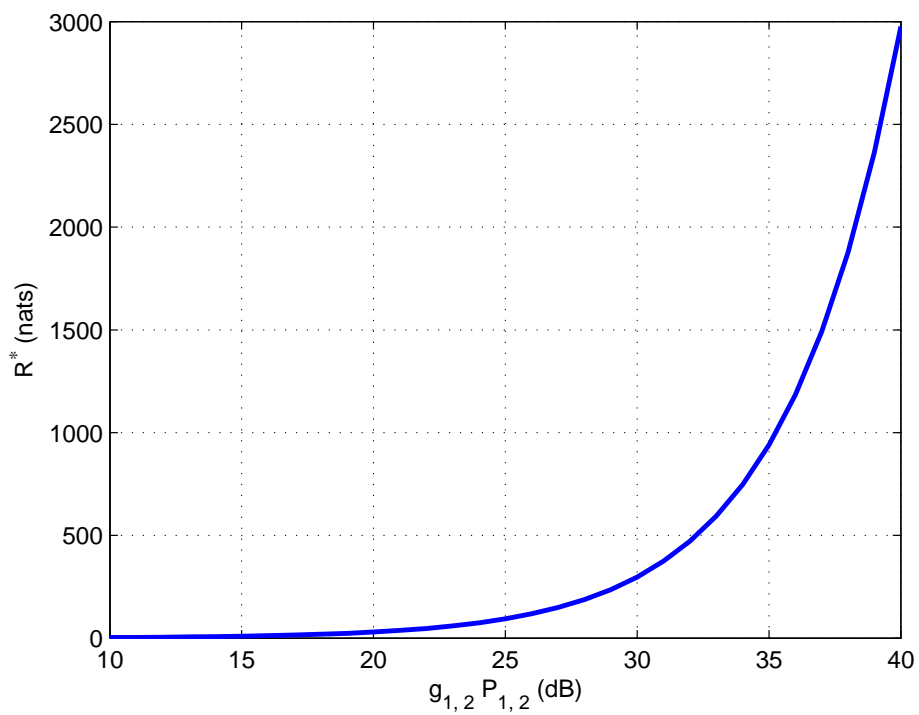

Fig. 3. The maximum achievable rate in wideband systems, as a function of hop links' SNR (both hop links have identical SNR in the plot).

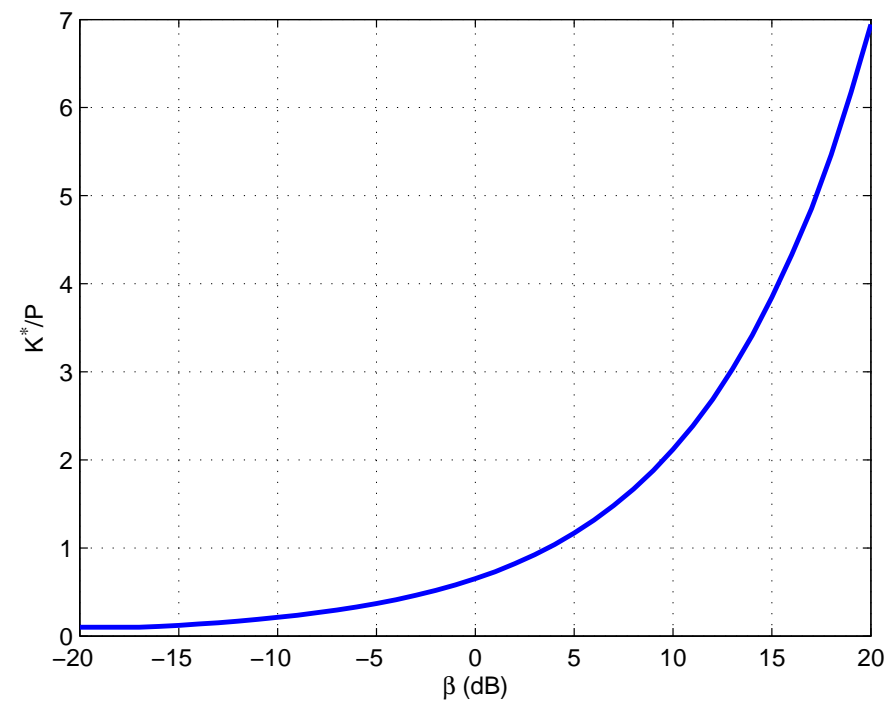

Fig. 4. The optimal number of active subcarriers (normalized by SNR of the source-relay hop link) as a function of $\beta$. 


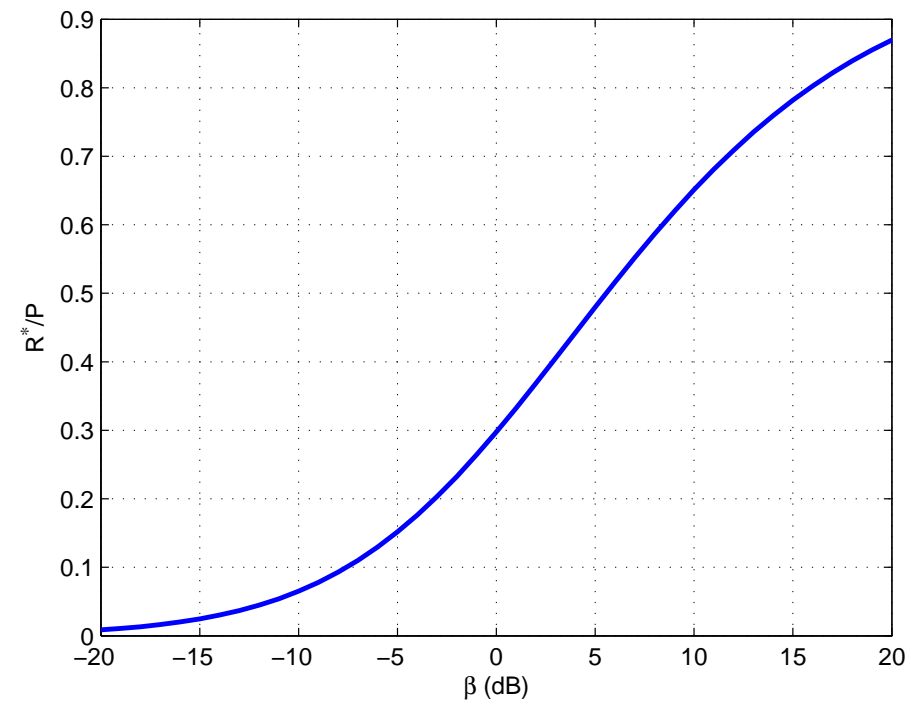

Fig. 5. The maximum achievable rate (normalized by SNR of the source-relay hop link) as a function of $\beta$.

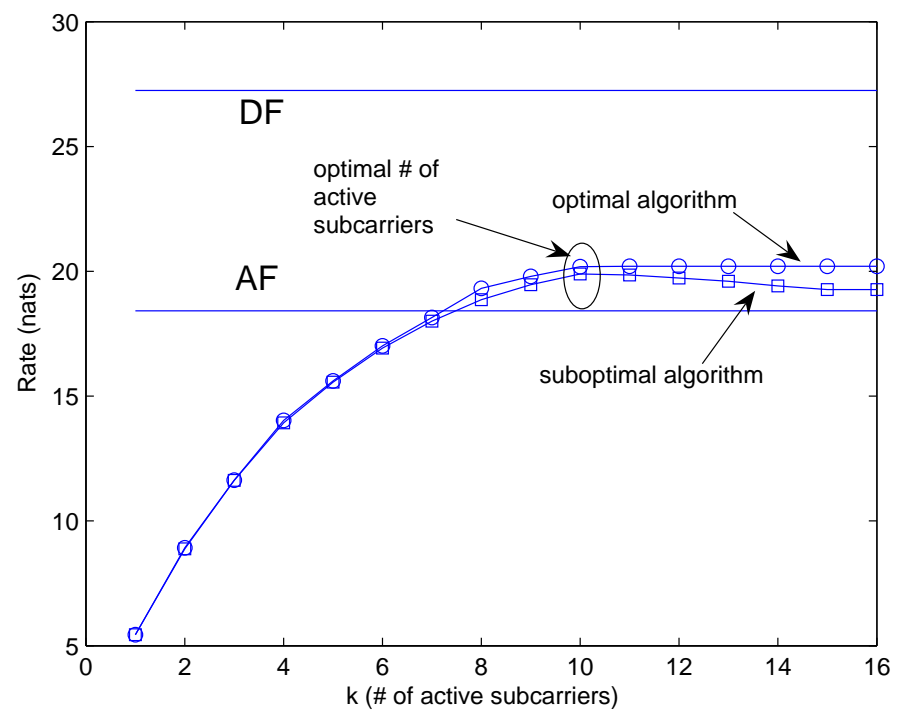

Fig. 6. The snapshot of the execution of the algorithms, corresponding to a particular channel realization sample. The system parameters are $N=16, P_{1}=P_{2}=20 \mathrm{~dB}$. 


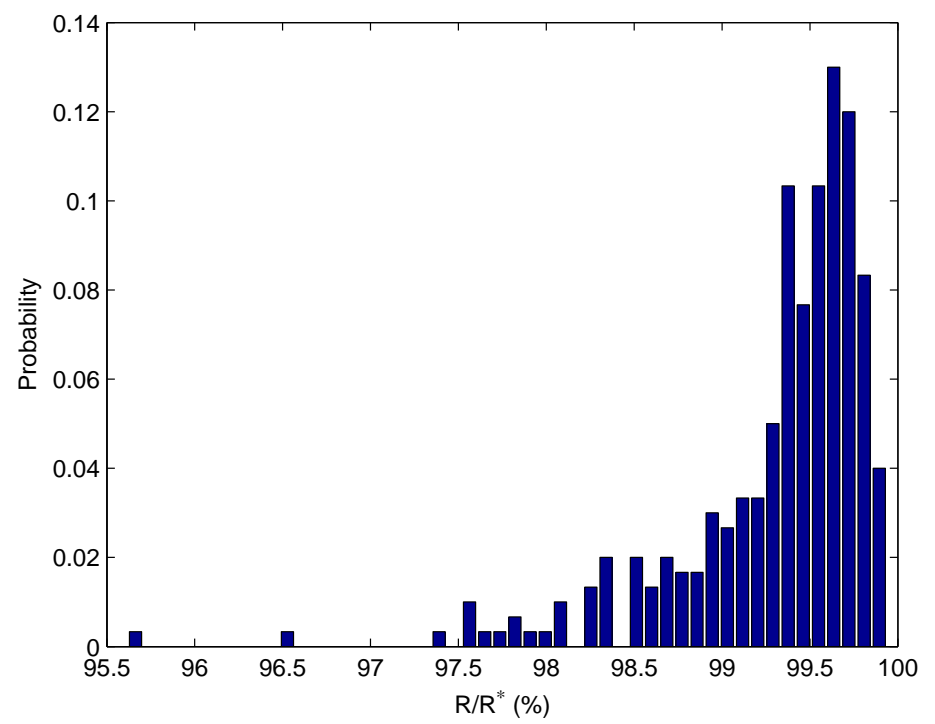

Fig. 7. The empirical histogram of the ratio between the rates achieved by the suboptimal algorithm and the optimal algorithm. The system parameters are $N=16, P_{1}=P_{2}=20 \mathrm{~dB}$. The number of channel realizations is 300 .

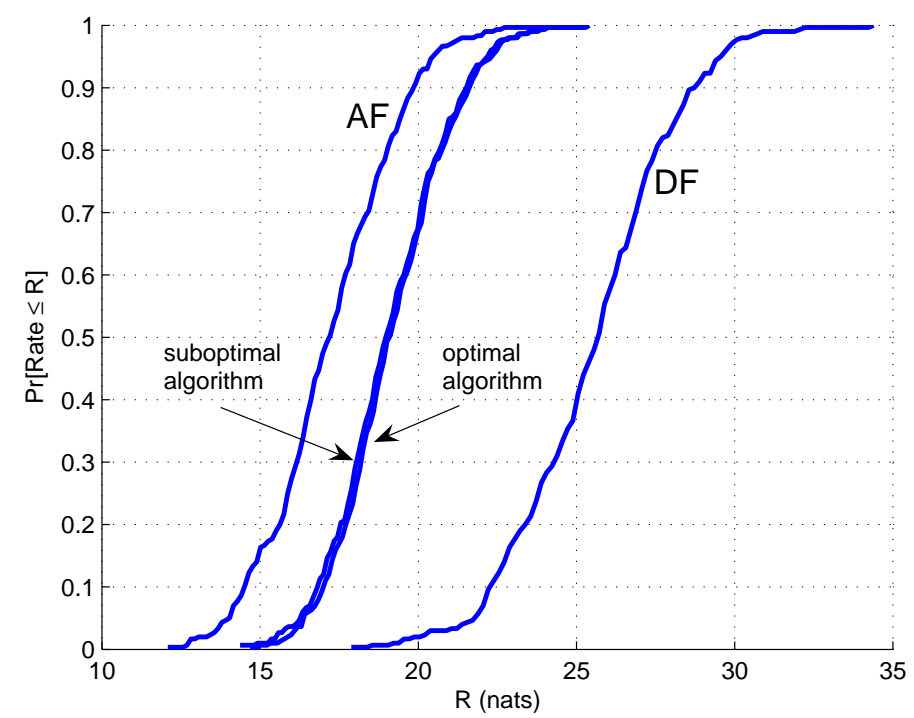

Fig. 8. The empirical CDF of the achievable rates. The system parameters are $N=16, P_{1}=P_{2}=20 \mathrm{~dB}$. The number of channel realizations is 300 . 


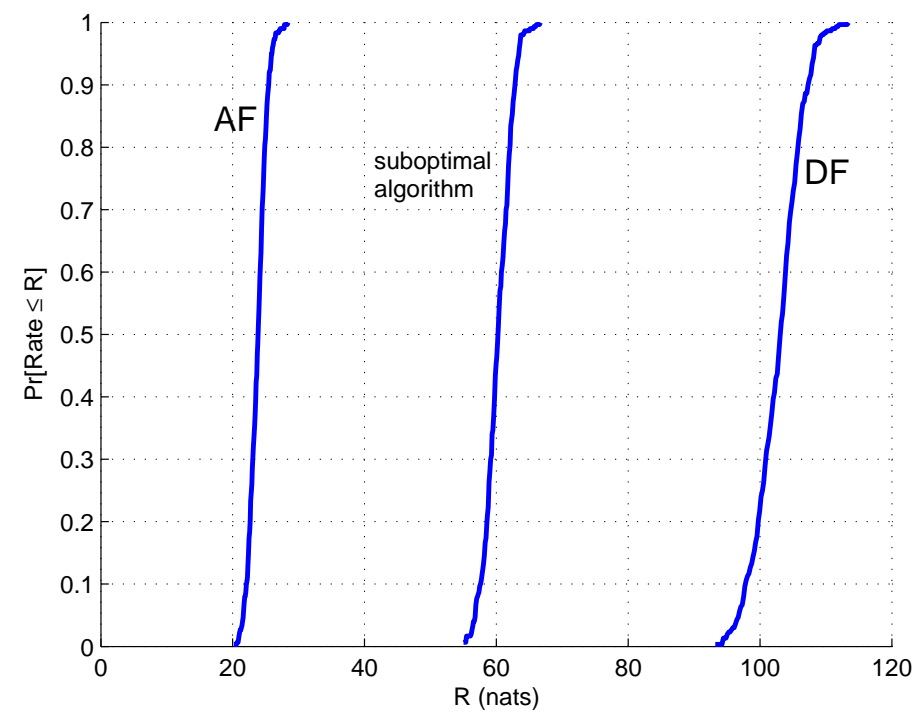

Fig. 9. The empirical CDF of the achievable rates. The system parameters are $N=256, P_{1}=P_{2}=20 \mathrm{~dB}$. The number of channel realizations is 300 . 